\title{
Aproximaciones al uso de los cursos en línea masivos y abiertos
}

\author{
Approaches to the Use of Massive Open Online Courses \\ Aproximacions a l'ús dels cursos en línia massius i oberts
}

\author{
Brenda Cecilia Padilla Rodríguez*i] \\ Universidad Autónoma de Nuevo León, Nuevo León, México \\ *Autor para correspondencia: brenda.padillardr@uanl.edu.mx (Brenda Cecilia Padilla Rodríguez)
}

| Publicado: 20/12/2019

RESUMEN: Desde su aparición en 2008, los cursos en línea masivos y abiertos (MOOCs, por sus siglas en inglés) han despertado interés a nivel internacional. El presente número de la revista Research in Education And Learning Innovation Archives (REALIA) se enfoca en distintas aproximaciones que se han dado al uso de este tipo de cursos. Sus temas abarcan las perspectivas de estudiantes, algunas cuestiones de diseño pedagógico y el desenvolvimiento de los participantes durante la implementación. Cada artículo incluido deja lecciones aprendidas y recomendaciones para futuros estudios. Así, este número de Research in Education And Learning Innovation Archives suma al conocimiento existente y marca la pauta para los siguientes pasos de investigación.

PALABRAS CLAVE: cursos en línea masivos y abiertos; MOOCs; editorial; diseño pedagógico; participación; estudiantes

Cómo citar: Padilla

Rodríguez, B. C. (2019). Aproximaciones al uso de los cursos en línea masivos y abiertos. Research in Education and Learning Innovation Archives, 23,40-42.

10.7203/realia.23.16293

Copyright: El/La Autor/a. Open Access: Este es un artículo de acceso abierto distribuido bajo los términos de la licencia Creative Commons AttributionNonCommercial-ShareAlike 4.0 International (CC BY-NC-SA 4.0)
ABSTRACT: Since their emergence in 2008, massive open online courses (MOOCs) have become an international source of interest. The current issue of Research in Education And Learning Innovation Archives (REALIA) focuses on different approaches to the use of this type of courses. Topics encompass students' perspectives, learning design and participants' performance during delivery. Each paper included leaves us with lessons learned and recommendations for future studies. Thus, this issue of Research in Education And Learning Innovation Archives adds to the existing knowledge in the field and sets the way for the next steps.

KEYWORDS: Massive Open Online Courses; MOOCs; editorial; course design; performance; students

RESUM: Des de la seua aparició en 2008, els cursos en línia massius i oberts (MOOCs, per les seues sigles en anglés) han despertat interés a nivell internacional. El present número de la revista Research in Education And Learning Innovation Archives (REALIA) s'enfoca en diferents aproximacions que s'han donat a l'ús d'aquesta mena de cursos. Els seus temes abasten les perspectives d'estudiants, algunes qüestions de disseny pedagògic i el desenvolupament dels participants durant la implementació. Cada article inclòs deixa lliçons apreses i recomanacions per a futurs estudis. Així, aquest número de Research in Education And Learning Innovation Archives suma al coneixement existent i marca la pauta per als següents passos d'investigació.

PARAULES CLAU: cursos en línia masius i oberts; MOOCs; editorial; disseny pedagògic; participació; alumnat 


\section{EDITORIAL}

Desde su aparición en 2008, los cursos en línea masivos y abiertos (MOOCs, por sus siglas en inglés) han despertado interés a nivel internacional (Johnson, Adams Becker, Estrada, y Freeman, 2015; Padilla Rodriguez, Bird, y Conole, 2015; Sharples et al., 2014). Su carácter usualmente gratuito los hace una opción formativa atractiva para estudiantes con perfiles variados que buscan explorar ambientes de educación en línea (e.g., Padilla Rodriguez et al., 2015), aprender sobre un tema en particular (e.g., Alario-Hoyos, Estévez-Ayres, Pérez-Sanagustín, Kloos, y Fernández-Panadero, 2017), o mejorar su desempeño laboral (e.g., Milligan y Littlejohn, 2014). Más de 100 millones de personas se han inscrito en al menos un MOOC (Shah y Pickard, 2019).

Desde el punto de vista institucional, los MOOCs pueden fungir como estrategias de mercadotecnia o administración, y contar con objetivos como el incremento de la visibilidad, el reclutamiento de estudiantes potenciales y la reducción de costos. También pueden servir para propósitos educativos, ya que al contar con una amplia audiencia representan un escenario ideal para experimentar con nuevas pedagogías y poner a prueba métodos y materiales didácticos (Hollands y Tirthali, 2014; Sharples et al., 2014). Más de 900 instituciones académicas y profesionales han desarrollado e implementado este tipo de cursos en plataformas especializadas, como Coursera, edX y MiriadaX (Shah y Pickard, 2019).

El presente número de la revista Research in Education And Learning Innovation Archives (REALIA) se enfoca en distintas aproximaciones que se han dado al uso de los MOOCs. Ballesteros-Ibarra, Mercado-Varela, y García-Vázquez (2019) describen las perspectivas de docentes de Sonora, México, quienes participaron en MOOCs para aprender sobre el nuevo modelo educativo nacional. En este trabajo se reafirma el valor de la formación en línea, pero también se identifican los retos que aún hoy en día siguen vigentes en ciertos contextos, como el temor a la tecnología. Se recomienda una colaboración entre autoridades educativas, diseñadores instruccionales y facilitadores de MOOCs para desarrollar estrategias para afrontar estos desafíos.

Por su parte, García, Barberà, y Maina (2019) estudian los procesos de regulación social del aprendizaje en los MOOCs. Analiza el uso de avisos (o prompts) orientadores, reflexivos y de retroalimentación como herramientas de andamiaje. Los primeros invitan a los participantes a actuar; los segundos estimulan estrategias metacognitivas; $y$ los terceros consisten en el reenvío automático de las respuestas a los avisos reflexivos. Los resultados son motivadores y conllevan implicaciones para la práctica en relación al diseño de MOOCs.

Finalmente, Mercado-Del-Collado, Jácome-Avila, Ortega-Guerrero, CasillasAlvarado, y Ramírez-Martinell (2019) revisan patrones de participación y logro en un MOOC de saberes digitales. Encuentran que los estudiantes de mayor edad y nivel educativo muestran un mejor desempeño. Asimismo enfatizan la tendencia de los MOOcs de seguir un modelo de embudo en donde la participación disminuye conforme avanzan las lecciones, y reflexionan sobre la necesidad de utilizar otras métricas de evaluación de efectividad.

Estos trabajos son sólo una pequeña muestra de la investigación que sigue siendo generada en relación a los MOOCs. Abarcan perspectivas de estudiantes, cuestiones de diseño pedagógico y desenvolvimiento de los participantes durante la implementación. Cada uno de ellos deja lecciones aprendidas y recomendaciones para futuros estudios. Así, este número de Research in Education and Learning Innovation Archives suma al conocimiento existente y marca la pauta para los siguientes pasos. Agradezco a todos los autores y espero que nuestros lectores lo disfruten. 


\section{REFERENCIAS}

Alario-Hoyos, C., Estévez-Ayres, I., Pérez-Sanagustín, M., Kloos, C. D., y Fernández-Panadero, C. (2017). Understanding Learners' Motivation and Learning Strategies in MOOCs. The International Review of Research in Open and Distributed Learning, 18(3).

https://doi.org/10.19173/irrodl.v18i3.2996

Ballesteros-Ibarra, M. L., Mercado-Varela, M. A., y García-Vázquez, N. J. (2019). La formación docente en línea: experiencias con MOOCs en Sonora (México). Research in Education And Learning Innovation Archives (REALIA), 23, 62-79.

https://doi.org/10.7203/realia.23.15903

García, I., Barberà, E., y Maina, M. (2019). Diseño de un sistema de apoyo a la regulación social del aprendizaje en los xMOOC. Research in Education and Learning Innovation Archives (REALIA), 23, 43-61. https://doi.org/10.7203/realia.23.15914

Hollands, F. M., y Tirthali, D. (2014). Why do institutions offer MOOCs. Fournal of Asynchronous Learning Network, 18, 1-20. Descargado de https://files.eric.ed.gov/fulltext/EJ1043160.pdf

Johnson, L., Adams Becker, S., Estrada, V., y Freeman, A. (2015). NMC Horizon Report: 2015 Higher Education Edition. Descargado de https://files.eric.ed.gov/fulltext/ED559357.pdf

Mercado-Del-Collado, R., Jácome-Avila, N., Ortega-Guerrero, J. C., Casillas-Alvarado, M. A., y Ramírez-Martinell, A. (2019). Patrones de participación y logro en un MOOC de Saberes Digitales para Docentes en servicio de México. Research in Education and Learning Innovation Archives (REALIA), 23, 80-95. https://doi.org/10.7203/realia.23.15904

Milligan, C., y Littlejohn, A. (2014). Supporting professional learning in a massive open online course. The International Review of Research in Open and Distributed Learning, 15(5). https://doi.org/10.19173/irrodl.v15i5.1855

Padilla Rodriguez, B. C., Bird, T., y Conole, G. (2015). Evaluation of Massive Open Online Courses (MOOCs): A Case Study. En T. Bastiaens y G. Marks (Eds.), Proceedings of Global Learn Berlin 2015: Global Conference on Learning and Technology (pp. 527-535). AACE. Descargado de https://www.learntechlib.org/primary/p/150900/

Shah, D., y Pickard, L. (2019, 30 de 07). Massive List of MOOC Providers Around The World. [Blog post]. Descargado de https://www.classcentral.com/report/mooc-providers-list/ Sharples, M., Adams, A., Ferguson, R., Gaved, M., Mcandrew, P., Rienties, B., ... Whitelock, D. (2014). Innovating Pedagogy 2014. Exploring new forms of teaching, learning and assessment, to guide educators and policy makers. Descargado de http://www.openuniversity.edu/sites/www.openuniversity.edu/files/ The_Open_University_Innovating_Pedagogy_2014_0.pdf 